OPEN ACCESS

Edited by:

Ming D. Li,

Zhejiang University, China

Reviewed by:

Maria Bove,

University of Foggia, Italy

Haijun Han,

Zhejiang University, China

${ }^{*}$ Correspondence:

Qi Wang

wangqi@gzucm.edu.cn

$\mathrm{Min} \mathrm{Li}$

doctorlimin@gzucm.edu.cn

Yousheng Mo

378480308@qq.com

${ }^{\dagger}$ These authors have contributed equally to this work

Specialty section: This article was submitted to Molecular Psychiatry, a section of the journal

Frontiers in Psychiatry

Received: 05 May 2021

Accepted: 09 July 2021

Published: 03 August 2021

Citation:

Duan L, Qiu W, Bai G, Qiao Y, Su S, Lo P-C, LU Y, Xu G, Wang Q, Li M and Mo Y (2021) Metabolomics Analysis on Mice With Depression Ameliorated

by Acupoint Catgut Embedding.

Front. Psychiatry 12:703516. doi: 10.3389/fpsyt.2021.703516

\section{Metabolomics Analysis on Mice With Depression Ameliorated by Acupoint Catgut Embedding}

\author{
Lining Duan 1,2t, Wenhui Qiu ${ }^{1,2 t}$, Guiqin Bai ${ }^{1}$, Yiqi Qiao ${ }^{1}$, Shiyu Su ${ }^{1,2}$, Po-Chieh Lo', \\ Yantong $\mathrm{Lu}^{1,3}$, Guofeng $\mathrm{Xu}^{4}$, Qi Wang ${ }^{1 *}$, Min $\mathrm{Li}^{1,2 *}$ and Yousheng Mo ${ }^{1,4 *}$ \\ ${ }^{1}$ Science and Technology Innovation Center, Guangzhou University of Chinese Medicine, Guangzhou, China, ${ }^{2}$ Clinical \\ Medical College of Acupuncture Moxibustion and Rehabilitation, Guangzhou University of Chinese Medicine, Guangzhou, \\ China, ${ }^{3}$ Guangdong Provincial People's Hospital, Guangzhou, China, ${ }^{4}$ The Second Affiliated Hospital of Guangzhou \\ University of Chinese Medicine, Guangzhou, China
}

Depression is a prevalent mental disease characterized by persistent low mood, lack of pleasure, and exhaustion. Acupoint catgut embedding (ACE) is a kind of modern acupuncture treatment, which has been widely used for the treatment of a variety of neuropsychiatric diseases. To investigate the effects and underlying mechanism of ACE on depression, in this study, we applied ACE treatment at the Baihui (GV20) and Dazhui (GV14) acupoints of corticosterone (CORT)-induced depression model mice. The results showed that ACE treatment significantly attenuated the behavioral deficits of depression model mice in the open field test (OFT), elevated-plus-maze test (EPMT), tail suspension test (TST), and forced swimming test (FST). Moreover, ACE treatment reduced the serum level of adreno-cortico-tropic-hormone (ACTH), enhanced the serum levels of 5hydroxytryptamine $(5-\mathrm{HT})$, and noradrenaline (NE). Furthermore, metabolomics analysis revealed that 23 differential metabolites in the brain of depression model mice were regulated by ACE treatment for its protective effect. These findings suggested that ACE treatment ameliorated depression-related manifestations in mice with depression through the attenuation of metabolic dysfunction in brain.

Keywords: acupoint catgut embedding, depression, corticosterone, brain, metabolomics

\section{INTRODUCTION}

Depression is a prevalent mental disease characterized by persistent low mood, lack of pleasure and exhaustion (1). According to the World Health Organization, more than 350 million individuals suffer from depression worldwide (2). More seriously, depression will become the second leading cause of disability by the year 2030 (3). At present, a combination of pharmacotherapy and psychological treatment is the preferable strategy for depression management (4). Unfortunately, the expensive treatment costs and intolerable adverse side effects greatly limit its clinical efficacy (5). Therefore, it is urgent to develop a safer and more effective method to treat depression.

Although, many hypotheses have been suggested, the exact pathogenesis of depression is still not well-understood (6). Hypothalamic-pituitary-adrenal (HPA) axis is a primary neuroendocrine axis which regulates the stress response in mammals (7). Chronic psychosocial stress can damage the negative feedback system of HPA axis and increase cortisol levels in patients with depression (8). High levels of cortisol increase the adrenocorticotrophic hormone (ACTH) synthesis and adversely affect the hippocampus, which play a key role in the processing of emotional stimuli 
and regulation of stress responses $(9,10)$. The dysfunction of HPA axis is an important feature of the pathophysiology of depression (11). Furthermore, increasing evidence suggests that metabolic abnormalities play an important role in the pathogenesis of depression (12-14). A recent systematic review on biomarkers for major depressive and bipolar disorders showed that the metabolic pathways served key roles in the pathophysiology of depression and they predominantly centered on glutamatergic metabolism, energy metabolism and neurotransmission $(15,16)$. Moreover, a clinical study demonstrated that polyunsaturated fatty acids metabolism, purine metabolism and inosine could be the potential independent diagnostic biomarkers for major depressive disorder (17). These findings strongly indicated that metabolic abnormalities may be a new target for depression diagnosis and treatment. Metabolomics is a novel biological approach which can comprehensively and quantitatively isolate and characterize the metabolites, whose changes can be considered as the ultimate response of body to physiological and pathological changes $(18,19)$. It has significant potential in the discovery of biomarkers for disease and the elucidation of therapeutic mechanisms $(20,21)$.

Acupuncture is a widespread therapy for depression which has been proven (22). A meta analysis including 2,268 participants demonstrated that acupuncture showed clinically significant reductions in the severity of depression. Moderate-quality evidence from a systematic review of 21 trails demonstrated that the overall risk of adverse events is higher with fluoxetine (FLX) or paroxetine than with acupuncture (23). Acupoint catgut embedding (ACE) is a kind of modern acupuncture treatment. Catgut is a type of absorbable surgical suture. It could be embedded in specific acupoints and the acupoints are stimulated continuously for 7-14 days until the catgut is absorbed by the body $(24,25)$. ACE treatment overcomes the insufficiency of acupuncture treatment such as long treatment cycle, frequent frequency and short stimulation time, and it has been widely used for the treatment of a variety of neuropsychiatric diseases $(26,27)$.

To investigate the effects and underlying mechanism of ACE on depression, in this study, we applied ACE treatment at the Baihui (GV20) and Dazhui (GV14) acupoints of corticosterone (CORT)-induced depression model mice. Behavior examination was conducted to evaluate the effect of ACE on depressivelike behaviors. Enzyme linked immunosorbent assay (ELISA) was conducted to determine the serum levels of ACTH, 5hydroxytryptamine (5-HT) and noradrenaline (NE). Liquid chromatography tandem mass spectrometry (LC-MS/MS)-based untargeted metabolomics analysis was performed to characterize the metabolic alterations in brain tissues. Our study provided new information on the effect and underlying mechanism of ACE in depression treatment.

\section{MATERIALS AND METHODS}

\section{Animal Groups}

A total of 80 male Swiss mice (20-25g, 7 weeks) were purchased from Guangdong Medical Laboratory Animal Center ( $<$ city $>$ Guangzhou $</$ city $>$, China). The mice were maintained with enough food and water at $24^{\circ} \mathrm{C}, 60 \%$ relative humidity and 12/12-h light/dark cycle. The mice were randomly divided into five groups ( $n=16 /$ group): control group (CON), CORT group (CORT), CORT with ACE treatment group (ACE), CORT with sham-ACE treatment group (SHAM), and CORT with fluoxetine (FLX) treatment group (FLX). FLX, a selective 5-HT reuptake inhibitor, is a first-line and widely used medication for depression. In this study, FLX was selected as a positive control to validate the efficacy of ACE treatment. CORT (Macklin, China) was dissolved in a saline solution containing $0.1 \%$ dimethyl sulfoxide and $0.3 \%$ Tween- 80 . To induce a depression model, the mice in the CORT, ACE, SHAM, and FLX groups received 20 $\mathrm{mg} / \mathrm{kg}$ CORT suspension daily at 9:00 am through subcutaneous injections for 21 consecutive days as previously described (28). While the mice in the CON group received an equal volumes of saline containing $0.1 \%$ dimethylsulfoxide and $0.3 \%$ Tween- 80 .

\section{Treatment}

All treatment measures were conducted $1 \mathrm{~h}$ later after CORT or saline administration. The mice in the ACE group were treated with ACE at GV20 and GV14 once on days 8 and 15. In mice, as in humans, the GV20 point is located at the intersection of the line linking the two mouse ear tips and the sagittal midline (29). The GV14 acupoint is located on the posterior midline and in the depression below the spinous process of the 7 th cervical vertebra in the prone position (30). ACE needles $0.6 \times$ $60 \mathrm{~mm}$ (Suzhou Medical Appliance) and Poly Lactic-co-Glycolic Acid (PLGA) absorbable sutures $0.2 \times 3 \mathrm{~mm}$ (Suzhou Medical Appliance) were used for the implantation. GV20 and GV14 acupoints were sterilized with $70 \%$ alcohol solution and the sutures were embedded at the $5 \mathrm{~mm}$ depth of acupoints using the ACE needles (Figure 1A). While the mice in the SHAM group were administrated with sham ACE treatment once on days 8 and 15. Briefly, GV20 and GV14 acupoints were inserted with the ACE needles to a depth of $5 \mathrm{~mm}$ without sutures implantation. FLX (Macklin, China) was dissolved in $0.9 \%$ sterile saline. The mice in the FLX group were treated with $15 \mathrm{mg} / \mathrm{kg}$ FLX once a day through oral gavage for 14 consecutive days (day 8-21). The mice in the CON and CORT groups received same grasping and fixing manipulation (Figure 1B).

\section{Behavioral Tests}

\section{Body Weight and Daily Food Intake Measurement}

In this study, the body weights and daily food intake of mice in each group were detected every week during the experimental period at 8 a.m.

\section{Open Field Test}

OFT was a well-established test to assess the locomotor activity, exploration habits and depression in the mice (31). OFT was performed in an open box structure $(50 \times 50 \times 40 \mathrm{~cm})$ with a black square at the bottom. The mouse was gently placed into the center of the square and allowed to move freely in the open field for $6 \mathrm{~min}$. The entire test consisted of $1 \mathrm{~min}$ of acclimation and $5 \mathrm{~min}$ of test. The percentage time in the center and the proportion traveled in the center were recorded during the 5min test period. After each mouse was tested, the open field 
A

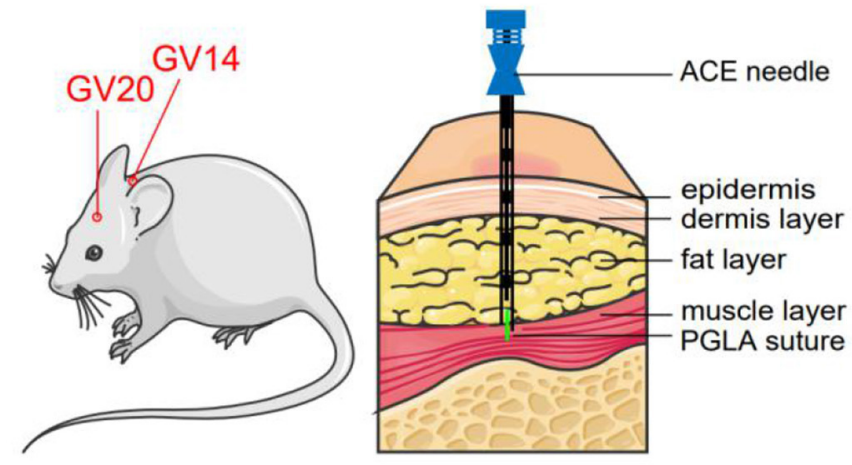

B

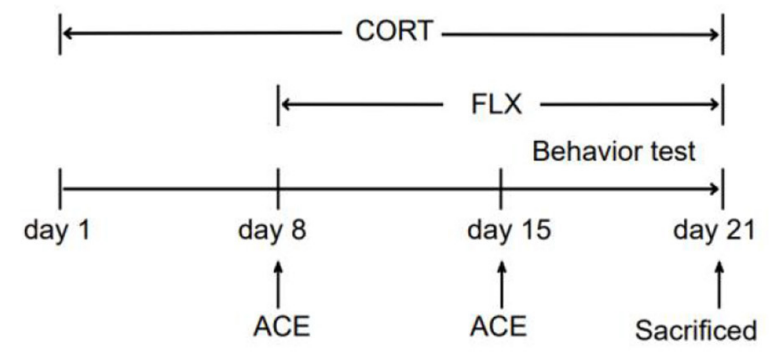

C

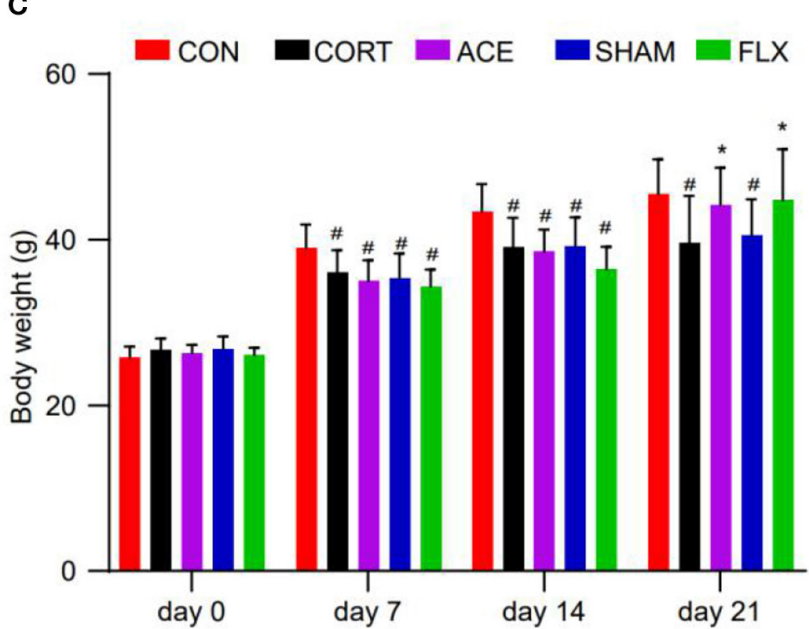

D

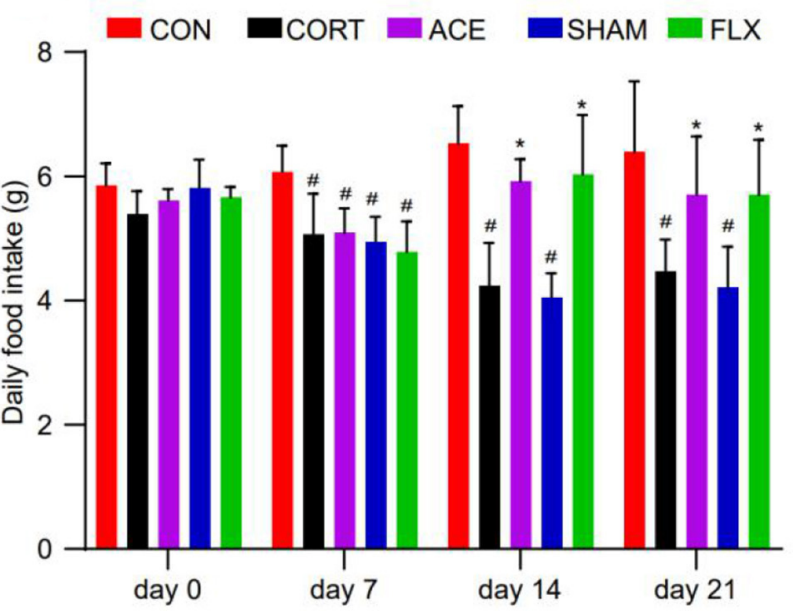

FIGURE 1 | Effect of ACE on the body weight and daily food intake of mice. (A) ACE treatment demonstrating the position of acupoints (GV20 and GV14) in mice. (B) Experimental schedule of this study. (C) The body weight of mice in all subgroups were weighted every week during the experimental period at 8 a.m. $(n=16$ per group). (D) The daily food intake of mice in all subgroups were weighted every week during the experimental period at 8 a.m ( $n=16$ per group). Experimental values were expressed as means \pm SD. ${ }^{*} P<0.05$ compared with the CON group. ${ }^{*} P 0.05$ compared with the CORT group.

was wiped and cleaned with $20 \%$ alcohol solution to eliminate smell interference.

\section{Elevated-Plus-Maze Test}

EPMT was used to evaluate the exploratory and anxietylike behaviors in mice (32). The elevated-plus maze for mice consisted of two perpendicular open arms $(35 \times 5 \mathrm{~cm})$ and two perpendicular closed arms $(35 \times 5 \times 20 \mathrm{~cm})$ connected by a central platform $(5 \times 5 \mathrm{~cm}), 50 \mathrm{~cm}$ above the floor. In this test, each mouse was placed at the center of maze and allowed to move freely in the maze for $6 \mathrm{~min}$. Each mouse was allowed to acclimate for $1 \mathrm{~min}$ before the test was started. The number of open arm entries (OE), the number of close arm entries (CE), the time spent in the open arm (OT), and the time spent in the close arm (CT) were recorded in the 5-min test session. The percent of time spent in the open arm $(\mathrm{OT} \%)=\mathrm{OT} /(\mathrm{OT}+\mathrm{CT}) \times 100 \%$. The percent of entries in the open arm $(\mathrm{OE} \%)=\mathrm{OE} /(\mathrm{OE}+\mathrm{CE}) \times 100 \%$. The maze was cleaned with a solution of $20 \%$ ethanol solution between the sessions.

\section{Tail Suspension Test}

TST is a test of behavioral despair and is commonly used to assess depressive-like behavior (33). In the TST, each mouse was suspended individually by the tail on the edge of a shelf placed $50 \mathrm{~cm}$ above a table. The mouse was secured in the place via adhesive tape positioned $\sim 0.5 \mathrm{~cm}$ from the tip of the tail. The duration of immobility was recorded for a test time of $5 \mathrm{~min}$. A video camera positioned in front of the animal was utilized to track the movement of each animal. The videorecords were analyzed using a software (Super Maze, Shanghai Xinruan Information Technology Co, Ltd., Shanghai, China) to make the trajectory diagrams of mice in TST.

\section{Forced Swimming Test}

FST was conducted to evaluate the despair-like behavior of mice in each group (34). In the test, the mouse was gently placed into a clear glass cylinder (diameter $11 \mathrm{~cm}$, height $30 \mathrm{~cm}$ ) containing $25^{\circ} \mathrm{C}$ water to a depth of $20 \mathrm{~cm}$. The duration of immobility was recorded during the last $5 \mathrm{~min}$ of the $6 \mathrm{~min}$ test session. Akin to 
TST, a video camera was placed in front of the animal to track the movement of each animal. The videorecords were analyzed by the software to make the trajectory diagrams of mice in FST.

\section{Serum Sample Preparation}

After behavioral analyses, the eyeballs of mice in each group were removed and the blood was collected. Blood samples were stood at room temperature for $2 \mathrm{~h}$ and then they were centrifuged at $3,500 \mathrm{rpm}$ for $15 \mathrm{~min}$ to separate serum. The serum samples were separated and stored in $\mathrm{a}-80^{\circ} \mathrm{C}$ freezer until analysis.

\section{ELISA Assay}

The ELISA kits of ACTH, 5-HT and NE were obtained from Jiangsu Meimian company (Nanjing, China). Serum ACTH, 5$\mathrm{HT}$, and NE levels of mice in each group were detected using the ELISA kits according to the instructions. The optical density was measured at $450 \mathrm{~nm}$ using a microplate reader (MULTISKAN EX, Thermoscientific). Serum ACTH, 5-HT, and NE levels were calculated from the standard curve.

\section{Metabolomics Analysis}

The metabolomics analysis work was completed by Shenzhen Huada Gene Technology Co., Ltd. (Shenzhen, China).

\section{Brain Tissue Samples Preparation}

After the behavioral experiments were finished, six mice from the CON group, CORT group and ACE group were anesthetized and then transcardially perfused with PBS. Brains were quickly removed, flash frozen in liquid nitrogen and stored at $-80^{\circ} \mathrm{C}$. Before detection, $25 \mathrm{mg}$ brain tissues were fixed with $800 \mu \mathrm{l}$ extraction buffer (methanol:acetonitrile:water $=2: 2: 1, \mathrm{v}: \mathrm{v}: \mathrm{v}$, $-20^{\circ} \mathrm{C}$ ). The samples were placed in a tissue grinding machine and ground for $5 \mathrm{~min}$ at $50 \mathrm{HZ}$. Afterwards, the samples were centrifuged at $25,000 \mathrm{rcf}$ for $15 \mathrm{~min}$ at $4^{\circ} \mathrm{C}$, and then the supernatant was removed. Next, a total of $600 \mu \mathrm{l}$ supernatant were dried with a vacuum concentration meter and added with reconstitution solution (methanol:water $=1: 9, \mathrm{v}: \mathrm{v}$ ). The samples were centrifuged again and the supernatant were placed in an autosampler vial for LC-MS/MS detection.

\section{LC-MS/MS Analysis}

Waters 2D UPLC system (Waters Corp., Milford, MA, USA) and Q Exactive tandem mass spectrometer (Thermo Fisher Scientific, USA) were used for metabolite separation and detection. Chromatographic separation was conducted using a $\mathrm{BEH} \mathrm{C} 18$ column $(1.7 \mu \mathrm{m} \mathrm{2.1*} 100 \mathrm{~mm}$, Waters, USA). The linear gradient programme set as follows: $2 \% \mathrm{~B}, 0-1 \mathrm{~min} ; 2-98 \% \mathrm{~B}, 1-9 \mathrm{~min} ; 98 \%$ B, 9-12 min; $98-2 \%$ B, $12.0-12.1$ min. $2 \%$ B, $12.1-15$ min. Flow rate was set $0.35 \mathrm{~mL} / \mathrm{min}$ and the column temperature was $45^{\circ} \mathrm{C}$. The injection volume was $5 \mu \mathrm{l}$.

MS data were collected with a Q Exactive tandem mass spectrometer (Thermo Fisher Scientific, USA). The mass spectra was a range of 70-1,050 mass-to-charge ratio with primary resolution of 70,000 and secondary resolution of 17,500. The ion source was electrospray ionization. The sheath gas flow rate was 40 and the aux gas flow rate was 10 . The capillary temp was $320^{\circ} \mathrm{C}$ and the aux gas heater temp was $350^{\circ} \mathrm{C}$.

\section{Data Analysis}

The data were processed using Compound Discoverer 3.0 (Thermo Fisher Scientific, USA), including peak alignment, peak extraction and compound identification. Partial least squares method-discriminant analysis (PLS-DA) was constructed and variable importance in projection (VIP) scores were calculated to estimate the importance of each variable in the PLS-DA projection. Univariate analysis and student's $t$-test were used for statistical analysis to evaluate the significant difference of potential biomarkers.

\section{Kyoto Encyclopedia of Genes and Genomes Enrichment Analysis}

The KEGG database was utilized to identify the relevant metabolic pathways and obtain a comprehensive metabolic bubble plot in the present work. In this study, KOBAS 2.0 software with the hyper-geometric test was used for KEGG pathway enrichment analysis. The differentially expressed metabolites file, the file including all the metabolites and the KEGG annotation file were input into the the software. The differentially expressed metabolites were set as foreground and all the metabolites were set as background. Functional enrichment analysis was estimated with the Fisher exact probability using the Gaussian hypergeometric test. KEGG pathways with a corrected $P$-value $<0.05$ were considered significantly enriched. Finally, a bubble plot for significant metabolic pathway enrichment analysis was established using the software.

\section{Statistical Analysis}

Experimental data were shown as mean \pm standard deviation (SD), and they were analyzed by the Statistical Package for the Social Sciences software (SPSS; version 25.0). Comparison between two groups was performed using $t$-tests. Comparisons between multiple groups were conducted using one way analysis of variance (ANOVA) followed by the least significant difference (LSD) test. The $P$-value $<0.05$ indicated statistical significance.

\section{RESULTS}

\section{Effect of ACE on the Body Weight and Daily Food Intake of CORT-Treated Mice}

Before the experiment, no difference was observed in body weight among the groups. Compared with the control, CORT administration obviously inhibited the body weight gain in mice. Compared with the CORT group, ACE and FLX treatment significantly promoted body weight gain. While sham-ACE treatment could not reverse the inhibitory effect of CORT on body weight increase (Figure 1C). Furthermore, compared with the CORT and SHAM groups, ACE and FLX treatment remarkably enhanced the daily food intake of mice (Figure 1D).

\section{Effect of ACE on CORT-Induced Depression-Like Behavior in Mice}

OFT test showed that ACE and FLX treatment markedly improved the percent of time spent in the center and the percent of distance in the center in comparison with CORT group. While sham-ACE treatment showed no significant effect 
on the percent of time spent in the center and the percent of distance in the center (Figures 2A-C). In the EPMT, ACE, and FLX treatment significantly enhanced the OT\% and OE\% of mice in relative to the CORT group and SHAM group. These results indicated that the administration of CORT suppressed the locomotor activity and spontaneous exploratory behavior of mice, while ACE and FLX treatment effectively reversed these effects (Figures 2D-F). TST test revealed that CORT administration significantly increased the immobility time of mice compared with the control. While ACE could reduce the immobility time to some degree (Figures 2G,H). Similarly, ACE and FLX treatment effectively decreased the immobility time in FST in relative to the CORT and SHAM group (Figures 2I,J). These results indicated that ACE treatment could ameliorate the CORT-induced despair behavior in mice.

\section{Effect of ACE on Serum ACTH, 5-HT, and NE Levels}

In this work, compared with the control, CORT administration significantly increased the serum ACTH levels of mice, while ACE and FLX treatment reduced the serum level of ACTH (Figure 3A). Moreover, the administration of CORT remarkably decreased the serum levels of 5-HT and NE. Nevertheless, ACE treatment increased the serum level of 5-HT to some degree (Figure 3B). Furthermore, ACE and FLX treatment significantly enhanced the serum levels of $\mathrm{NE}$ in the depression mice. Nevertheless, sham-ACE treatment showed no significant effect (Figure 3C).

\section{Metabolomics Analysis of Brain Tissues}

The metabolic profiles of brain samples from the CON, CORT and ACE groups were characterized by LC-MS/MS. The analysis detected a total of 1,684 ion compounds.

\section{PLS-DA Analysis}

To obtain the detailed metabolic differences between the CON, CORT and ACE groups, the data were subjected to PLS-DA analysis. Significant differences between the CON and CORT groups were observed in PLS-DA score plot, indicating that the metabolic profiles were dramatically altered following CORT administration. In contrast, the remarkable differences between the CORT and ACE groups in PLS-DA score plot suggested that ACE treatment lead to a significant callback effect on metabolic profiles (Figures 4A,B). The PLS-DA model was validated using the response of the permutation test through 200 permutations, in which all R2 were close to 1 and Q2 were lower than 0 . The good PLS-DA model indicated an excellent predictive power (Figures 4C,D).

\section{Differential Metabolites Screening}

In our present work, the differential metabolites screening conditions were set as following: The VIP of two principal components for the PLS-DA model were $\geq 1$. Fold-Change $\geq 1.2$ or $\leq 0.83$. $P$-value $<0.05$ (Table 1 ). The results showed that a total of 181 metabolites were significantly and differently expressed in CORT mice compared with the control, with 155 down-regulated metabolites and 26 upregulated metabolites (Figure 5A). A total of 162 metabolites in the ACE mice relative to the CORT mice displayed significantly differential expression, with 89 metabolites upregulated and 73 metabolites down-regulated (Figure 5B). ACE treatment reversed 21 down-regulated metabolites induced by CORT administration (Figure 5C). Additionally, ACE treatment reversed two up-regulated metabolites (glycerin, spermidine) induced by CORT administration (Figure 5D). These findings suggested that the behaviors of depression in the mice were significantly attenuated via the intervention of ACE, which might be achieved through regulating the above differential metabolites in brain. These differential metabolites may be the treatment targets for ACE to exert its anti-depressive effects.

\section{Metabolic Pathway Analysis}

The results of KEGG enrichment analysis showed that metabolic pathway was significantly changed after ACE treatment. There are seven differential metabolites located in metabolic pathway. Furthermore, the regulation of leucine, valine and methionine affect the biosynthesis of amino acids, 2-Oxocarboxylic acid metabolism, mineral absorption and protein digestion, and absorption. In addition, the up-regulation of leucine after ACE treatment regulated the mTOR signaling pathway. Moreover, ACE treatment significantly regulated retrograde endocannabinoid signaling in the brain tissues of depression mice. Additionally, ABC transporters, Aminoacyl-tRNA biosynthesis and other energy signaling pathways might be also involved in the beneficial effects of ACE against depression (Figure 6).

\section{DISCUSSION}

In this study, we established a depression mice model as previously described through CORT repeated administration (28). Chronic CORT administration can mimic the chronic stress associated with the dysfunction of HPA axis in rodents (35). Consistent with previous studies, in this research, the CORTinduced depression mice showed behavioral and neurochemical alterations, which are the characteristics of depression.

Traditional Chinese medicine (TCM) believes that GV20 and GV14 both belong to the Governor Vessel, may function to collect the energy from peripheral regions and transport it to the brain, thus, energizing the entire body (36). GV20 is strongly associated with the brain, and acupuncture at GV20 can regulate cerebral functions (37). GV14 is the convergence of the Governor Vessel with all yang meridians (38). According to the theory of TCM, the synergistic and complementary effects of different acupoints compatibility supposedly have an advantage in clinical practice to enhance the curative efficacy and reduce the side effects (39). In clinical practice, GV20 and GV14 are usually used in combination with each other (40). Previous studies demonstrated that GV20 and GV14 are optimized acupoints combinations for neuropsychiatric disorders, which could improve the cerebral blood circulation, enhance memory, and improve antidepressant activity, playing a key role in brain protection $(41,42)$. Importantly, a clinical study reported that ACE treatment on GV20 and GV14 can effectively regulate the anxiety and depressive symptoms of patients with gastroesophageal reflux disease (43). So, in our 
A

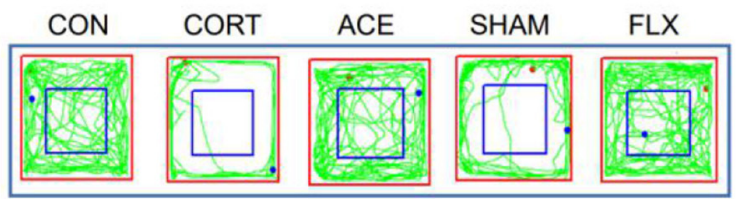

B

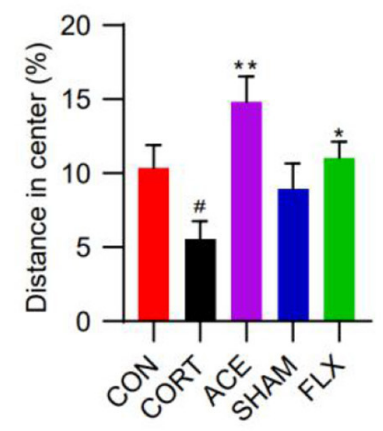

G

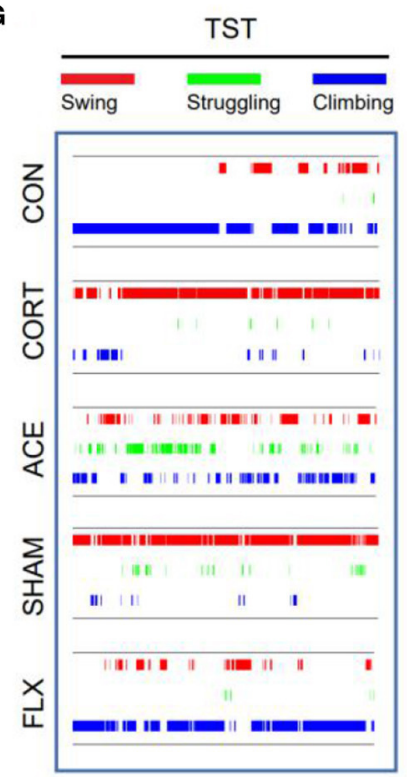

C

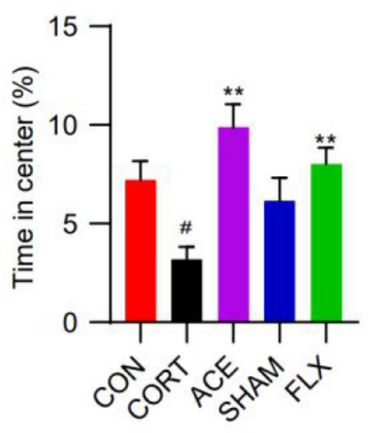

I

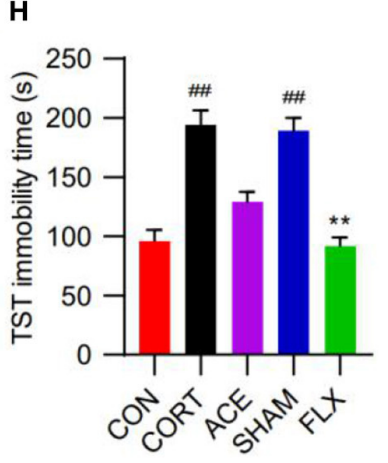

D

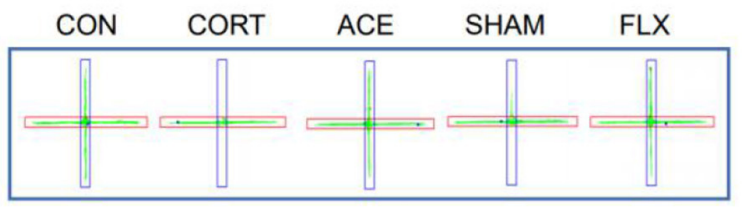

E

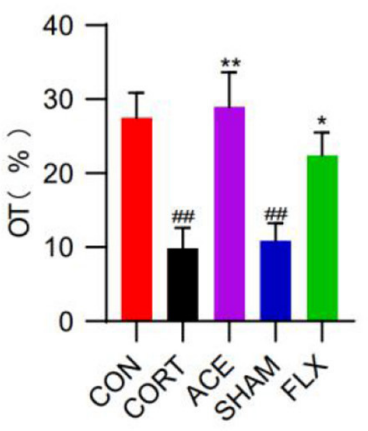

F

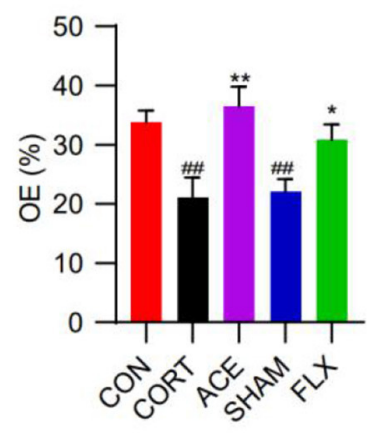

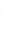
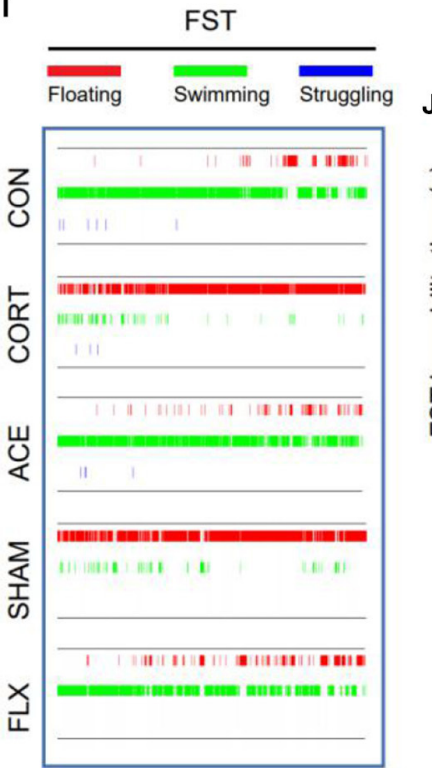

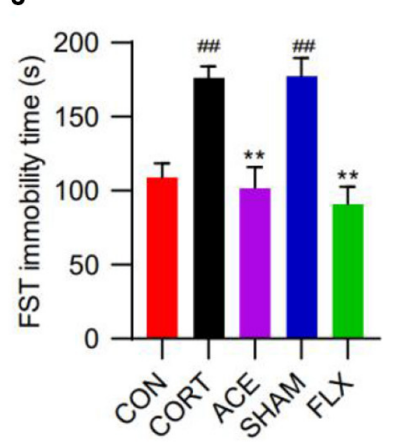

FIGURE 2 | Effect of ACE on the depression-like behavioral deficits of mice. (A) The representative trajectory diagrams of mice in OFT. (B) ACE significantly increased the distance in center of mice in OFT ( $n=16$ per group). (C) ACE significantly increased the time in center of mice in OFT ( $n=16$ per group). (D) The representative trajectory diagrams of mice in EPMT. (E) ACE significantly increased the OT\% of mice in EPMT ( $n=16$ per group). (F) ACE significantly increased the OE\% of mice in EPMT ( $n=16$ per group). (G) The representative trajectory diagrams of mice in TST. (H) ACE decreased the immobility of mice in TST to some degree without statistical significance ( $n=16$ per group). (I) The representative trajectory diagrams of mice in FST. (J) ACE significantly decreased the immobility of mice in FST ( $n=$ 16 per group). Experimental values were expressed as means \pm SD. ${ }^{\#} P<0.05$ and ${ }^{\# \#} P<0.01$ compared with the CON group. ${ }^{\star} P<0.05$ and ${ }^{* *} P<0.01$ compared with the CORT group.

work, we investigated the efficacy of ACE treatment at both GV20 and GV14 acupoints on depression model mice. We found that ACE treatment effectively revised the depressive-like behavior in the mice, suggesting that the characteristic presentations of depression could be reversed by ACE treatment. Notably, CORT administration lead to the dysfunction of HPA axis as evident from the marked increase in the serum level of ACTH.
However, ACE treatment effectively mitigated the dysfunction of HPA axis as indicated by the reduction in serum ACTH level. Furthermore, ACE treatment reversed the decrease of serum 5-HT and NE levels caused by CORT administration. These results strongly indicated that ACE could improve depressive-like behaviors and regulate neurotransmitter release in depression model mice. 

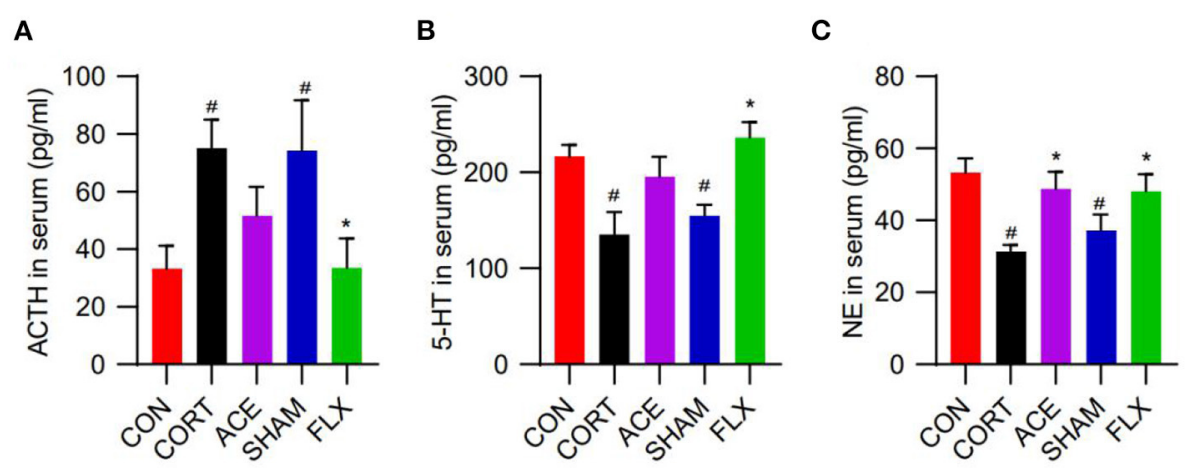

FIGURE 3 | Effect of ACE on the serum neurotransmitter levels of mice. (A) ACE decreased the serum ACTH level of mice to some degree without statistical significance ( $n=8$ per group). (B) ACE increased the serum $5-\mathrm{HT}$ level of mice to some degree without statistical significance $(n=8$ per group). (C) ACE significantly increased the serum NE level of mice ( $n=8$ per group). Experimental values were expressed as means $\pm \mathrm{SD}$. $\# P<0.05$ compared with the CON group. $P<0.05$ compared with the CORT group.

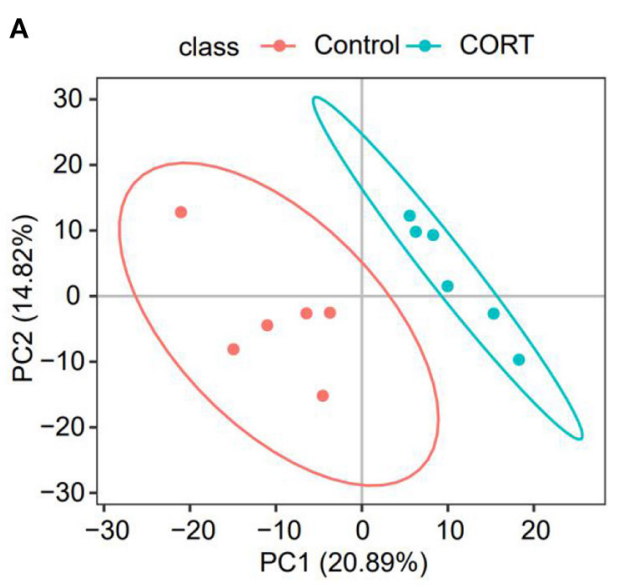

C

Intercepts: $\mathrm{R} 2=(0.0,0.98), \mathrm{Q} 2=(0.0,-0.65)$

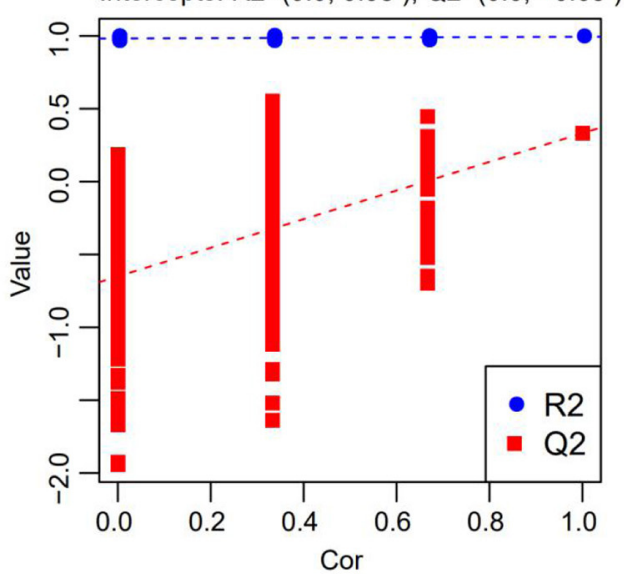

B

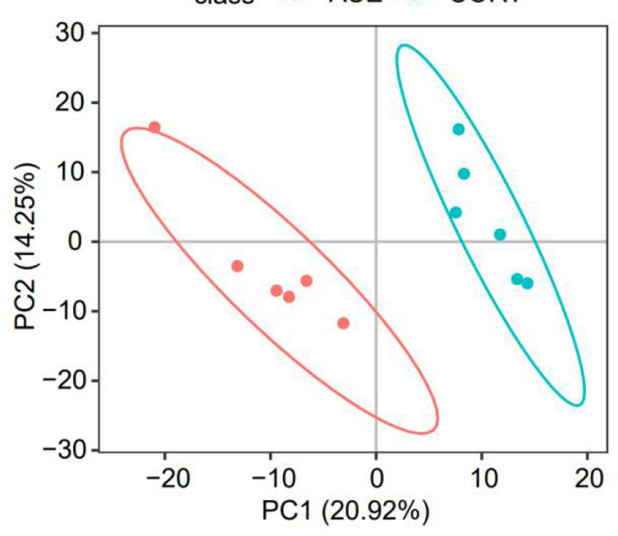

D

Intercepts: $\mathrm{R} 2=(0.0,0.99), \mathrm{Q} 2=(0.0,-0.66)$

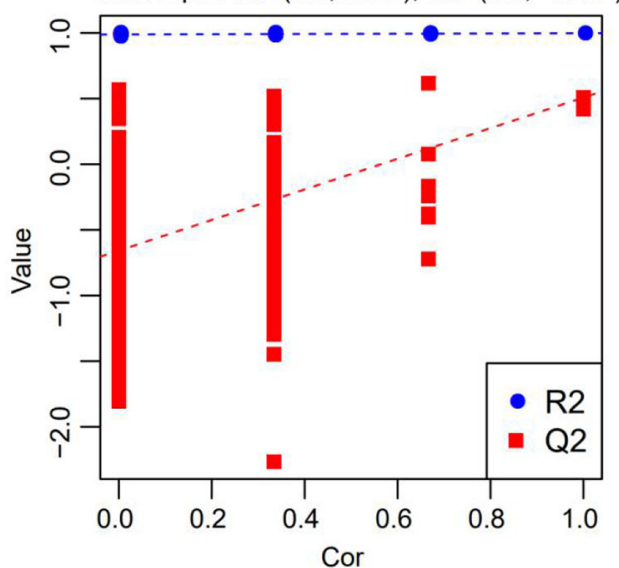

FIGURE 4 | PLS-DA analysis of the samples from the CON, CORT and ACE groups. (A,B) Score graph of the PLS-DA analysis model. The horizontal axis is the first principal component, and the vertical axis is the second principal component. The number in parentheses is the score of the principal component, which represents the percentage of the explanation on overall variance of the specific pricipal component. ( $n=6$ per group) (C,D) PLS-DA analysis model response permutation testing graph. The two rightmost points in the figure are the actual R2Y and Q2 values of the model, and the remaining points are the R2Y and Q2 values obtained by randomly arranging the samples used. This result is mainly used to judge whether the model is overfit and the validity of the model ( $n=6$ per group). 
TABLE 1 | A total of 23 differential metabolites in brain were significantly regulated by ACE treatment for its protective effect $(\mathrm{VIP} \geq 1$, Fold-Change $\geq 1.2$ or $\leq 0.83$, $p$-value <0.05).

\begin{tabular}{|c|c|c|c|c|}
\hline Name & Structural formula & Value & Fold-change & $\begin{array}{l}\text { variable importance in } \\
\text { projection }\end{array}$ \\
\hline Atagabalin & $\mathrm{C} 10 \mathrm{H} 19 \mathrm{NO} 2$ & 0.006015343 & 2.030789853 & 2.437886173 \\
\hline NA & $\mathrm{C} 10 \mathrm{H} 22 \mathrm{~N} 2 \mathrm{O} 4$ & 0.044708202 & 2.939875336 & 2.373943613 \\
\hline To0127900 & $\mathrm{C} 9 \mathrm{H} 17 \mathrm{NO}$ & 0.001059841 & 1.626339433 & 2.361939214 \\
\hline NA & $\mathrm{C} 18 \mathrm{H} 31 \mathrm{NO} 2$ & 0.002237832 & 1.705992477 & 2.237393434 \\
\hline Glycine & $\mathrm{C} 8 \mathrm{H} 15 \mathrm{NO} 7$ & 0.001126475 & 1.616287438 & 2.029389852 \\
\hline Butyryl-I-homosErine lactone & $\mathrm{C} 8 \mathrm{H} 13 \mathrm{NO} 3$ & 0.043026322 & 1.610630149 & 1.895197878 \\
\hline Linoleamide & $\mathrm{C} 18 \mathrm{H} 33 \mathrm{NO}$ & 0.000921998 & 1.477077597 & 1.885952314 \\
\hline NA & $\mathrm{C} 15 \mathrm{H} 19 \mathrm{NO} 5$ & 0.025102575 & 1.588355158 & 1.878871577 \\
\hline Tyrosine & $\mathrm{C} 12 \mathrm{H} 16 \mathrm{~N} 2 \mathrm{O} 4$ & 0.042120026 & 1.678155724 & 1.862066732 \\
\hline Leucine & $\mathrm{C} 6 \mathrm{H} 13 \mathrm{NO} 2$ & 0.047073181 & 1.582969519 & 1.84846739 \\
\hline Hymexazol o-glucoside & $\mathrm{C} 10 \mathrm{H} 15 \mathrm{NO} 7$ & 0.032752292 & 1.595695617 & 1.756172084 \\
\hline NA & C9H7N5 & 0.034528607 & 1.538238734 & 1.733662313 \\
\hline$(+)$-castanospermine & $\mathrm{C} 8 \mathrm{H} 15 \mathrm{NO} 4$ & 0.029853408 & 1.482080518 & 1.687325726 \\
\hline Spiroxamine & $\mathrm{C} 18 \mathrm{H} 35 \mathrm{NO} 2$ & 0.002198635 & 1.300628861 & 1.397939511 \\
\hline Valine & $\mathrm{C} 10 \mathrm{H} 20 \mathrm{~N} 2 \mathrm{O} 3$ & 0.012587926 & 1.474244747 & 1.387117836 \\
\hline $\mathrm{N}$-acetyl-I-2-aminoadipic acid & C8H13NO5 & 0.040711557 & 1.361018957 & 1.359001501 \\
\hline NA & $\mathrm{C} 9 \mathrm{H} 15 \mathrm{NO} 5$ & 0.011348663 & 1.367427359 & 1.345577446 \\
\hline Retinol & $\mathrm{C} 2 \mathrm{OH} 30 \mathrm{O}$ & 0.021802618 & 1.223678168 & 1.049871149 \\
\hline 2-[(5z,8z,11z)-icosatrienoyl]-sn-glycero-3-phosphoethanolamine & $\mathrm{C} 25 \mathrm{H} 46 \mathrm{NO} 7 \mathrm{P}$ & 0.026806339 & 2.77159524 & 1.01498837 \\
\hline Testosterone & $\mathrm{C} 19 \mathrm{H} 28 \mathrm{O} 2$ & 0.019892157 & 5.437394848 & 3.669634374 \\
\hline Glycerin & $\mathrm{C} 23 \mathrm{H} 38 \mathrm{O} 4$ & 0.014765512 & 0.763749521 & 2.381849619 \\
\hline Spermidine & $\mathrm{C} 9 \mathrm{H} 21 \mathrm{~N} 3 \mathrm{O}$ & 0.025804694 & 0.795712175 & 3.65936226 \\
\hline Cysteine & $\mathrm{C} 3 \mathrm{H} 7 \mathrm{NO} 2 \mathrm{~S}$ & 0.072097044 & 1.702991174 & 2.279302811 \\
\hline
\end{tabular}

To further determine the mechanism of ACE on depression, we found 23 differential metabolites regulated by ACE treatment in the brain for its positive effect. It is well-known that the neurotransmitters are intimately associated with depression (44). Amino acids are the precursors of these neurotransmitters and depleted levels of them are observed in depression patients (45). Moreover, amino acids metabolism has the potential for predicting the therapeutic response in depression. Importantly, amino acids supplements could alleviate the symptoms of depression (46). Leucine, valine and tyrosine are necessary precursors for the synthesis neurotransmitters. Leucine plays a pivotal role in a variety of physiological and pathological processes, including neuronal function, immunity and aging (47, 48). An integrated meta-analysis demonstrated that the level of leucine was significantly reduced in patients with major depressive disorder (16). Moreover, leucine and valine participate in the formation of 5-HT (49). The low concentration of 5HT in the synaptic cleft has been implicated as a main etiology of depression (50). Consistent with previous studies, in our present work, we found that the levels of leucine and valine were decreased in the brain tissues of the CORT group, while ACE treatment significantly reversed these effects. Moreover, tyrosine, as an aromatic amino acid, is a precursor for the synthesis of catecholamine neurotransmitters $(51,52)$. It was reported that tyrosine was essential in neurotransmitters synthesis under the catalysis of tyrosine hydroxylase $(53,54)$. It should be noted that the pathogenesis of depression is associated with catecholamine neurotransmitters synthesis disturbances, such as dopamine and NE $(55,56)$. In this work, ACE treatment effectively attenuated the CORT-induced tyrosine reduction in the brain tissues.

Glycine is a type of protective agent, which has been reported to have antioxidant and anti-inflammatory activities in vivo (57). Previous works demonstrated that glycine could suppress oxidative stress, neuroinflammation, and apoptotic neurodegeneration in postnatal rat brain $(47,58)$. Cysteine possesses both anti-oxidant and anti-inflammatory properties (59). Importantly, glutathione, as a tripeptide that functions as an antioxidant, which were formed from cysteine and glycine (60). In this study, ACE significantly enhanced the cysteine and glycine levels in mice with depression, which indicated its neuroprotective efficacy through antioxidant and anti-inflammation mechanisms.

In addition, ACE decreased the levels of glycerin and spermidine in the brain tissues of depression model mice. A large-scale meta-analysis showed that the disorder of lipid metabolism is strongly associated with depression (13). In agreement with previous works, we found that the amount of glycerin was remarkably increased in depression mice. In contrast, ACE effectively reduced the glycerin level $(61,62)$. Spermidine could promote autophagic activation, which may induce neuronal damage in the hippocampus $(61,62)$. ACE administration effectively suppressed the level of spermidine 

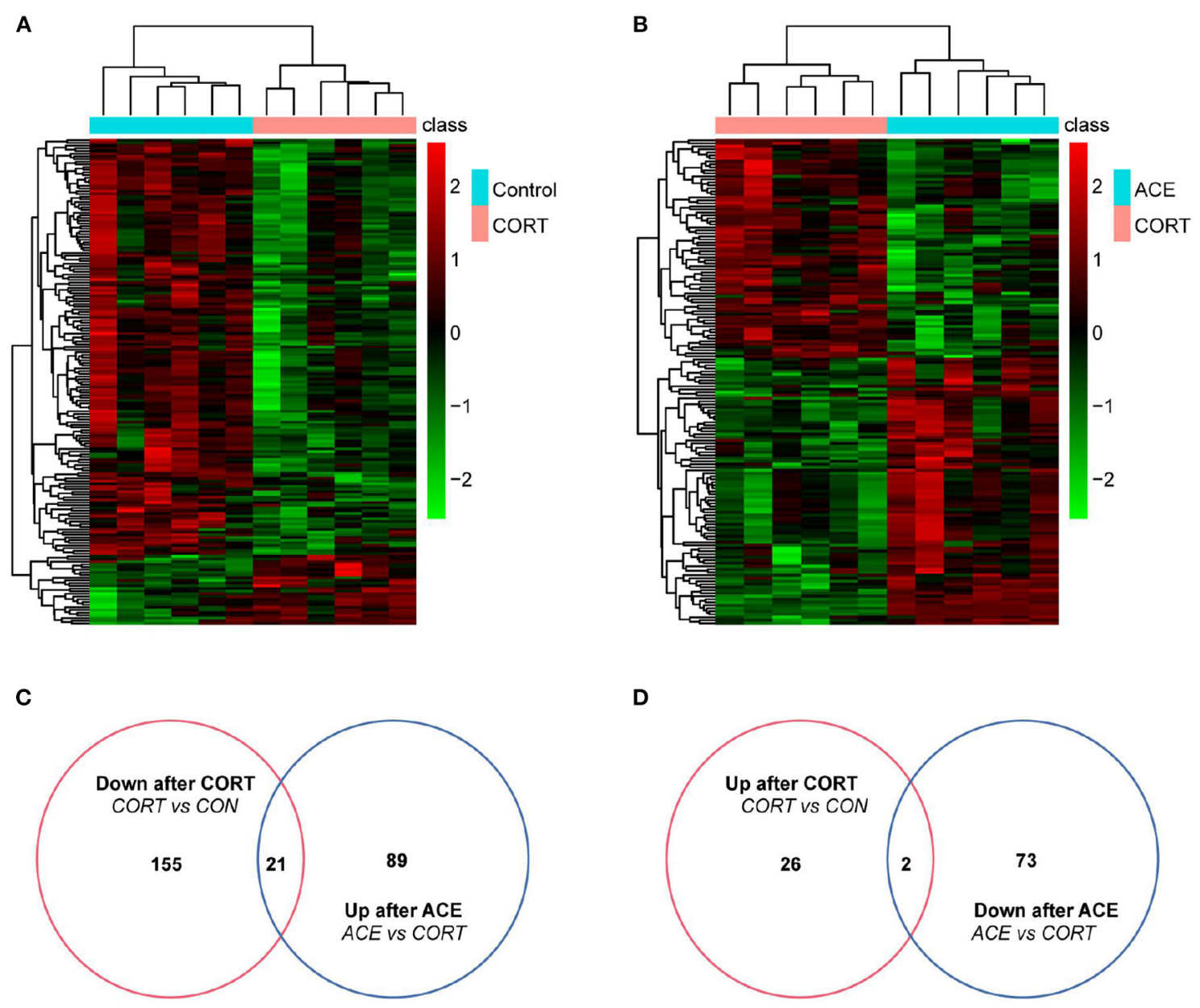

FIGURE 5 | Differential metabolites expression in the brain of mice between the CON, CORT, and ACE groups. (A) Heatmap of differentially expressed metabolites between CON and CORT samples with green and red spectrum colors indicating downregulated and upregulated expression, respectively $(p<0.05, n=6$ per group). (B) Heatmap of differentially expressed metabolites between ACE and CORT samples with green and red spectrum colors indicating downregulated and upregulated expression, respectively ( $p<0.05, n=6$ per group). (C,D) Venn diagrams showed the overlaps of differentially expressed metabolites between experimental groups. A total of 21 metabolites decreased in CORT group but increased in ACE group. A total of 2 metabolites increased expression in CORT group but decreased in ACE group.

in the brain. Additionally, it was found that ACE significantly enhanced the contents of testosterone in the brain. Resent study demonstrated that testosterone could prevent depression through MAPK/ERK2 signaling pathway (63). Linoleamide is an important active ingredient of Maca, which could regulate the energy metabolism and increased the antioxidant capacity (64).

Antioxidant vitamins play an important role in the physiological processes including neuroprotection, oxidative free radical production and immune-modulatory functions. Clinical studies revealed that the serum levels of vitamin A, E, and $\mathrm{C}$ were significantly decreased in patients with depression (65). The pathophysiological processes of depression are closely related to oxidative stress and many studies reported that some neurological diseases could be prevented by antioxidant vitamins treatments (66). In this work, it was found that ACE treatment inhibited CORT-induced decrease of retinol (vitamin A). These findings suggested that ACE might exert its therapeutic effects on depression through its antioxidant activities. Taken together, these results suggested that ACE treatment had a notably modulatory effect on the dysregulated cerebral metabolism in the mice with depression. To date, no studies have addressed the alterations of tagabalin, butyryl-l-homos erine lactone, hymexazol o-glucoside, castanospermine, and spiroxamine during depression. These metabolites might has potential in diagnosis and treatment of depression. Herein our results provide evidence on perturbations in the cerebral metabolites of depression.

The results of KEGG enrichment analysis showed that metabolic pathway was significantly changed after ACE treatment. There are seven differential metabolites located in metabolic pathway including glycerin, leucine, valine, pyridoxamine, methionine, testosterone, and retinol. These 


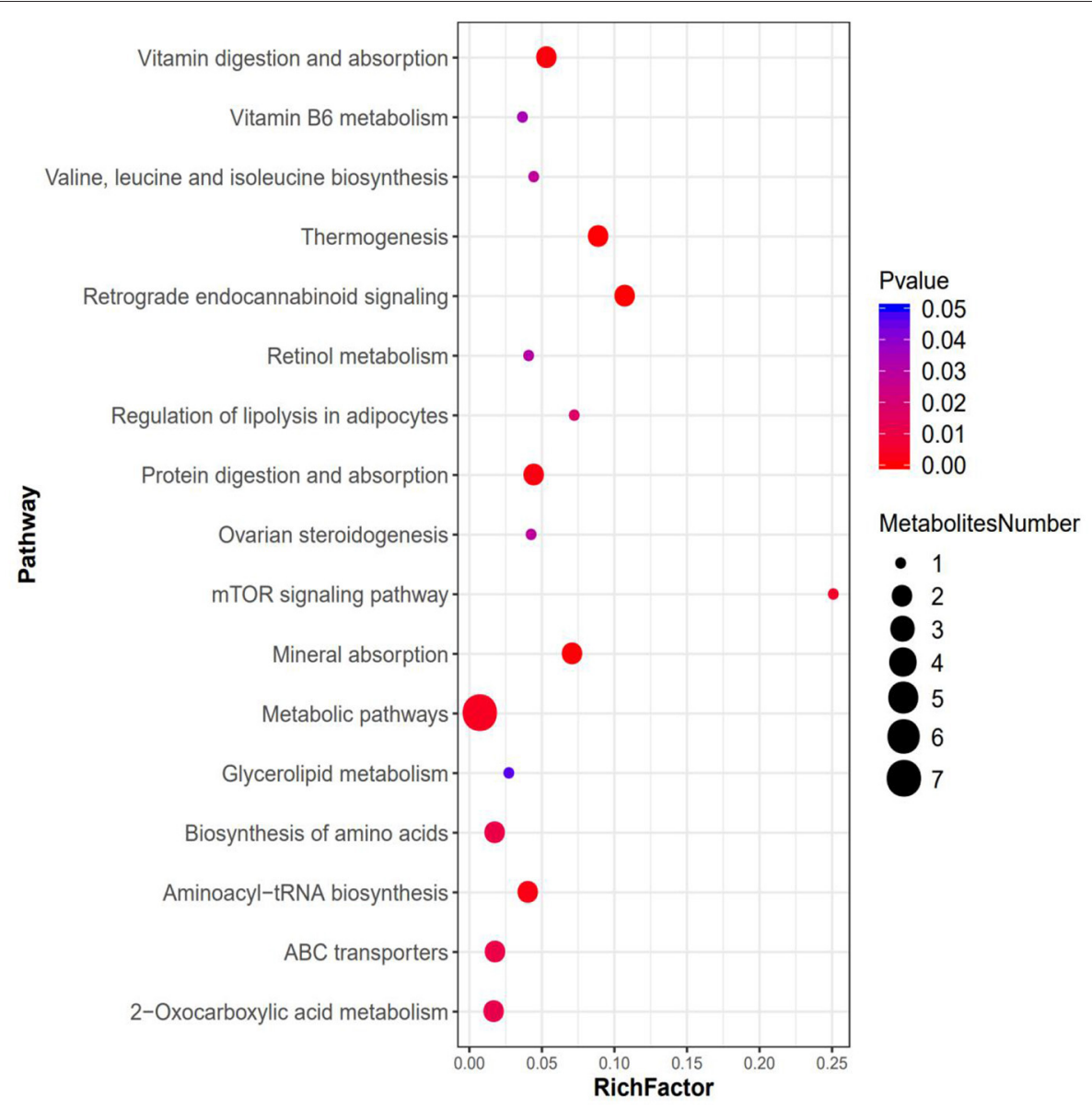

FIGURE 6 | Bubble plots for metabolic pathway enrichment analysis between the ACE and CORT group ( $n=6$ per group). X-axis enrichment factor (RichFactor) is the number of differential metabolites annotated to the pathway divided by all identified metabolites annotated to the pathway. The larger the value, the greater the proportion of differential metabolites annotated to the pathway. The dot size represents the number of differential metabolites annotated to this pathway.

findings strongly suggested that ACE treatment could regulate amino acid metabolism, lipid metabolism, and vitamin metabolism in depression. Furthermore, the regulation of leucine, valine, and methionine also affect the biosynthesis of amino acids, 2-Oxocarboxylic acid metabolism, mineral absorption, and protein digestion and absorption. In addition, it was suggested that leucine could activate mTOR signaling pathway, which has a protective role in synaptic plasticity in stress and depression $(67,68)$. In this study, KEGG results revealed that the up-regulation of leucine after ACE treatment regulated the mTOR signaling pathway in depression mice. Moreover, endocannabinoids could regulate mood, emotion, appetite, pain, and cognition via the stimulation of cannabinoid receptors. And depression is associated with the decrease in retrograde endocannabinoid signaling in the hippocampus (69). In this work, it was found that ACE treatment significantly regulated retrograde endocannabinoid signaling in the brain tissues of depression mice. Additionally, KEGG analysis demonstrated that $\mathrm{ABC}$ transporters, Aminoacyl-tRNA biosynthesis and other energy signaling pathways might be also involved in the beneficial effects of ACE against depression. These results suggest that the integrated regulation of ACE on multipathways and multitargets.

\section{CONCLUSION}

In conclusion, our study revealed that ACE treatment significantly attenuated the depressive-like behaviors and regulated neurotransmitter release in CORT-induced depression model mice. Importantly, the metabolomics analysis showed that 23 differential metabolites in brain were regulated by ACE treatment for its protective effect. These findings strongly suggested that ACE treatment could ameliorate depressionrelated manifestations through the attenuation of metabolic dysfunction in brain.

\section{DATA AVAILABILITY STATEMENT}

The original contributions presented in the study are included in the article/supplementary 
material, further inquiries can be directed to the corresponding author/s.

\section{ETHICS STATEMENT}

The animal study was reviewed and approved by the Laboratory Animal Ethics Committee of Guangzhou University of Chinese Medicine.

\section{AUTHOR CONTRIBUTIONS}

LD and WQ designed and conducted the study with equal contribution. GB, YQ, and SS contributed to the implementation of the experiments. P-CL and YL contributed

\section{REFERENCES}

1. Barnett R. Depression. Lancet. (2019) 393:2113. doi: 10.1016/S0140-67 36(19)31151-1

2. Kuehn BM. Postpartum depression screening needs more consistency. JAMA. (2020) 323:2454. doi: 10.1001/jama.2020.9737

3. Wormser GP, Hassett AL. Depression in the primary care setting. $N$ Engl J Med. (2019) 380:2278-9. doi: 10.1056/NEJMc1903259

4. Munkholm K, Jørgensen KJ, Paludan-Müller AS. Continuing antipsychotic medication for patients with psychotic depression in remission. JAMA. (2019) 322:2443. doi: 10.1001/jama.2019.1 7691

5. Bertocci MA, Hanford L, Manelis A, Iyengar S, Youngstrom EA, Gill MK, et al. Clinical, cortical thickness and neural activity predictors of future affective lability in youth at risk for bipolar disorder: initial discovery and independent sample replication. Mol Psychiatry. (2019) 24:185667. doi: 10.1038/s41380-018-0273-4

6. Zimmerman M, Morgan TA, Stanton K. The severity of psychiatric disorders. World Psychiatry. (2018) 17:258-75. doi: 10.1002/wps.20569

7. Hamada M, Nishigawa $T$, Maesono S, Aso $K$, Ikeda $H$, Furuse $M$. Decreased stress-induced depression-like behavior in lactating rats is associated with changes in the hypothalamic-pituitary-adrenal axis, brain monoamines, and brain amino acid metabolism. Stress. (2019) 22:48291. doi: 10.1080/10253890.2019.1584179

8. Bleker LS, van Dammen L, Leeflang M, Limpens J, Roseboom TJ, de Rooij SR. Hypothalamic-pituitary-adrenal axis and autonomic nervous system reactivity in children prenatally exposed to maternal depression: a systematic review of prospective studies. Neurosci Biobehav Rev. (2020) 117:24352. doi: 10.1016/j.neubiorev.2018.05.033

9. Geerlings MI, Gerritsen L. Late-life depression, hippocampal volumes, and hypothalamic-pituitary-adrenal axis regulation: a systematic review and meta-analysis. Biol Psychiatry. (2017) 82:339-50. doi: 10.1016/j.biopsych.2016.12.032

10. Wu T, Huang Y, Gong Y, Xu Y, Lu J, Sheng H, et al. Treadmill exercise ameliorates depression-like behavior in the rats with prenatal dexamethasone exposure: the role of hippocampal mitochondria. Front Neurosci. (2019) 13:264. doi: 10.3389/fnins.2019.00264

11. Labad J, Soria V, Salvat-Pujol N, Segalàs C, Real E, Urretavizcaya $\mathrm{M}$, et al. Hypothalamic-pituitary-adrenal axis activity in the comorbidity between obsessive-compulsive disorder and major depression. Psychoneuroendocrinology. (2018) 93:208. doi: 10.1016/j.psyneuen.2018.04.008

12. Malhi GS, Mann JJ. Depression. Lancet. (2018) 392:2299312. doi: 10.1016/S0140-6736(18)31948-2

13. Bot M, Milaneschi Y, Al-Shehri T, Amin N, Garmaeva S, Onderwater G, et al. Metabolomics profile in depression: a pooled analysis of 230 metabolic markers in 5283 cases with depression and 10,145 controls. Biol Psychiatry. (2020) 87:409-18. doi: 10.1016/j.biopsych.2019.08.016 to the formal analysis and data curation. GX revised the manuscript. QW, ML, and YM supervised the study. All authors contributed to the article and approved the submitted version.

\section{FUNDING}

This work was supported by Key Laboratory Project of Colleges and Universities in Guangdong Province (No. 2019KSYS005), Guangzhou Science Technology and Innovation Commission Technology Research Projects (No. 201805010005), and Guangdong Province Science and Technology Plan International Cooperation Project (No. 2020A0505100052).

14. Nedic Erjavec G, Sagud M, Nikolac Perkovic M, Svob Strac D, Konjevod M, Tudor L, et al. Depression: biological markers and treatment. Prog Neuropsychopharmacol Biol Psychiatry. (2021) 105:110139. doi: 10.1016/j.pnpbp.2020.110139

15. Ogawa S, Koga N, Hattori K, Matsuo J, Ota M, Hori H, et al. Plasma amino acid profile in major depressive disorder: analyses in two independent case-control sample sets. J Psychiatr Res. (2018) 96:2332. doi: 10.1016/j.jpsychires.2017.09.014

16. MacDonald K, Krishnan A, Cervenka E, Hu G, Guadagno E, Trakadis Y. Biomarkers for major depressive and bipolar disorders using metabolomics: a systematic review. Am J Med Genet B Neuropsychiatr Genet. (2019) 180:12237. doi: 10.1002/ajmg.b.32680

17. Zhou X, Liu L, Lan X, Cohen D, Zhang Y, Ravindran AV, et al. Polyunsaturated fatty acids metabolism, purine metabolism and inosine as potential independent diagnostic biomarkers for major depressive disorder in children and adolescents. Mol Psychiatry. (2019) 24:147888. doi: 10.1038/s41380-018-0047-z

18. Hashimoto K. Metabolomics of major depressive disorder and bipolar disorder: overview and future perspective. Adv Clin Chem. (2018) 84:8199. doi: 10.1016/bs.acc.2017.12.005

19. Stapelberg N, Pratt R, Neumann DL, Shum D, Brandis S, Muthukkumarasamy $\mathrm{V}$, et al. From feedback loop transitions to biomarkers in the psychoimmune-neuroendocrine network: detecting the critical transition from health to major depression. Neurosci Biobehav Rev. (2018) 90:1-15. doi: 10.1016/j.neubiorev.2018.03.005

20. Savitz J. Role of kynurenine metabolism pathway activation in major depressive disorders. Curr Top Behav Neurosci. (2017) 31:249-67. doi: 10.1007/7854_2016_12

21. Song J, Ma W, Gu X, Zhao L, Jiang J, Xu Y, et al. Metabolomic signatures and microbial community profiling of depressive rat model induced by adrenocorticotrophic hormone. J Transl Med. (2019) 17:224. doi: 10.1186/s12967-019-1970-8

22. Armour M, Smith CA, Wang LQ, Naidoo D, Yang GY, MacPherson H, et al. Acupuncture for depression: a systematic review and meta-analysis. J Clin Med. (2019) 8:1140. doi: 10.3390/jcm8081140

23. Qaseem A, Barry MJ, Kansagara D. Non-pharmacologic versus pharmacologic treatment of adult patients with major depressive disorder: a clinical practice guideline from the american college of physicians. Ann Intern Med. (2016) 164:350-9. doi: 10.7326/M15-2570

24. Huang F, Xie Y, Zhao S, Feng Z, Chen G, Xu Y. The effectiveness and safety of acupoint catgut embedding for the treatment of postmenopausal osteoporosis: a systematic review and meta-analysis. Evid Based Complement Alternat Med. (2019) 2019:2673763. doi: 10.1155/2019/2673763

25. Zhang Y, Gong H, Zhan B, Chen S. Acupoint catgut embedding reduces insulin resistance in diabetic patients undergoing open cardiac surgery. Heart Surg Forum. (2021) 24:E060-4. doi: 10.1532/hsf.3331

26. Xu F, Xuan LH, Zhou HJ, Chen FY, Zheng ZJ, Bi Y, et al. Acupoint catgut embedding alleviates insomnia in different chinese medicine syndrome 
types: a randomized controlled trial. Chin J Integr Med. (2019) 25:5439. doi: $10.1007 / \mathrm{s} 11655-018-2770-3$

27. Wang YH, Chen WT. Foreign body cystic granuloma and abscess after acupoint catgut embedding. Br J Dermatol. (2021) 185:e1. doi: 10.1111/bjd.20056

28. Kv A, Madhana RM, Js IC, Lahkar M, Sinha S, Naidu V. Antidepressant activity of vorinostat is associated with amelioration of oxidative stress and inflammation in a corticosterone-induced chronic stress model in mice. Behav Brain Res. (2018) 344:73-84. doi: 10.1016/j.bbr.2018.02.009

29. Frantz AL, Regner GG, Pflüger P, Coelho VR, da Silva LL, Viau CM, et al. Manual acupuncture improves parameters associated with oxidative stress and inflammation in PTZ-induced kindling in mice. Neurosci Lett. (2017) 661:33-40. doi: 10.1016/j.neulet.2017.09.044

30. Jung YS, Lee SW, Park JH, Seo HB, Choi BT, Shin HK. Electroacupuncture preconditioning reduces ROS generation with NOX4 down-regulation and ameliorates blood-brain barrier disruption after ischemic stroke. J Biomed Sci. (2016) 23:32. doi: 10.1186/s12929-016-0249-0

31. Kurosawa N, Shimizu K, Seki K. The development of depression-like behavior is consolidated by IL-6-induced activation of locus coeruleus neurons and IL-1 $\beta$-induced elevated leptin levels in mice. Psychopharmacology. (2016) 233:1725-37. doi: 10.1007/s00213-015-4084-x

32. Lo Iacono L, Ielpo D, Accoto A, Di Segni M, Babicola L, D\&\#39, et al. MicroRNA-34a regulates the depression-like behavior in mice by modulating the expression of target genes in the dorsal raphè. Mol Neurobiol. (2020) 57:823-36. doi: 10.1007/s12035-019-01750-2

33. Li W, Liu X, Qiao H. Downregulation of hippocampal SIRT6 activates AKT/CRMP2 signaling and ameliorates chronic stressinduced depression-like behavior in mice. Acta Pharmacol Sin. (2020) 41:1557-67. doi: 10.1038/s41401-020-0387-5

34. Taniguchi E, Tashiro A, Hattori A, Furuse M, Yasuo S. Photoperiodic changes in hippocampal neurogenesis and plasma metabolomic profiles in relation to depression-like behavior in mice. Behav Brain Res. (2021) 403:113136. doi: 10.1016/j.bbr.2021.113136

35. Oliveira TQ, de Sousa C, Vasconcelos GS, de Sousa LC, de Oliveira AA, Patrocínio C, et al. Brain antioxidant effect of mirtazapine and reversal of sedation by its combination with alpha-lipoic acid in a model of depression induced by corticosterone. J Affect Disord. (2017) 219:4957. doi: 10.1016/j.jad.2017.05.022

36. Chi L, Du K, Liu D, Bo Y, Li W. Electroacupuncture brain protection during ischemic stroke: a role for the parasympathetic nervous system. J Cereb Blood Flow Metab. (2018) 38:479-91. doi: 10.1177/0271678X17697988

37. Lu J, Liu X, Tian Y, Li H, Ren Z, Liang S, et al. Moxibustion exerts a neuroprotective effect through antiferroptosis in parkinson's disease. Evid Based Complement Alternat Med. (2019) 2019:2735492. doi: 10.1155/2019/2735492

38. Hou L, Gu F, Gao G, Zhou C. Transcutaneous electrical acupoint stimulation (TEAS) ameliorates chemotherapy-induced bone marrow suppression in lung cancer patients. J Thorac Dis. (2017) 9:809-17. doi: 10.21037/jtd.2017.03.12

39. Chen B, Hu SX, Liu BH, Zhao TY, Li B, Liu Y, et al. Efficacy and safety of electroacupuncture with different acupoints for chemotherapy-induced nausea and vomiting: study protocol for a randomized controlled trial. Trials. (2015) 16:212. doi: 10.1186/s13063-015-0734-x

40. Zhang R, Cai X, Song X, Dong C, Hou X, Lv L. Normalization of ventral tegmental area structure following acupuncture in a rat model of heroin relapse. Neural Regen Res. (2014) 9:301-7. doi: 10.4103/1673-5374.128228

41. Ahn SM, Kim YR, Kim HN, Shin YI, Shin HK, Choi BT. Electroacupuncture ameliorates memory impairments by enhancing oligodendrocyte regeneration in a mouse model of prolonged cerebral hypoperfusion. Sci Rep. (2016) 6:28646. doi: 10.1038/srep 28646

42. Zheng Y, He J, Guo L, Yao L, Zheng X, Yang Z, et al. Transcriptome analysis on maternal separation rats with depression-related manifestations ameliorated by electroacupuncture. Front Neurosci. (2019) 13:314. doi: 10.3389/fnins.2019. 00314

43. Luo Z, Hu X, Chen C, Zhu L, Zhang W, Shen Y, et al. Effect of catgut embedment in du meridian acupoint on mental and psychological conditions of patients with gastroesophageal reflux disease. Evid Based Complement Alternat Med. (2020) 2020:5415813. doi: 10.1155/2020/5415813
44. Nagasawa M, Ogino Y, Kurata K, Otsuka T, Yoshida J, Tomonaga S, et al. Hypothesis with abnormal amino acid metabolism in depression and stress vulnerability in Wistar Kyoto rats. Amino Acids. (2012) 43:210111. doi: $10.1007 /$ s00726-012-1294-y

45. Woo HI, Chun MR, Yang JS, Lim SW, Kim MJ, Kim SW, et al. Plasma amino acid profiling in major depressive disorder treated with selective serotonin reuptake inhibitors. CNS Neurosci Ther. (2015) 21:41724. doi: $10.1111 / \mathrm{cns} .12372$

46. Lakhan SE, Vieira KF. Nutritional therapies for mental disorders. Nutr J. (2008) 7:2. doi: 10.1186/1475-2891-7-2

47. Duan DD, Wang KX, Zhou YZ, Qin XM, Gao L, Du GH. Baicalein exerts beneficial effects in d-galactose-induced aging rats through attenuation of inflammation and metabolic dysfunction. Rejuvenation Res. (2017) 20:50616. doi: 10.1089/rej.2017.1919

48. Walker AK, Wing EE, Banks WA, Dantzer R. Leucine competes with kynurenine for blood-to-brain transport and prevents lipopolysaccharideinduced depression-like behavior in mice. Mol Psychiatry. (2019) 24:152332. doi: 10.1038/s41380-018-0076-7

49. Ellul P, Gasnier M, Trebossen V, Gaillard R. Immune activation and deficit in neurotransmitters synthesis in treatment resistant depression: about a case of hashimoto encephalopathy. Clin Psychopharmacol Neurosci. (2020) 18:463-6. doi: 10.9758/cpn.2020.18.3.463

50. Zhang $\mathrm{H}$, Huang $\mathrm{H}$, Song $\mathrm{H}$, Chin $\mathrm{B}$, Zheng $\mathrm{H}$, Ruan $\mathrm{J}$, et al. Serum metabolomics reveals the intervention mechanism and compatible regularity of Chaihu Shu Gan San on chronic unpredictable mild stressinduced depression rat model. J Pharm Pharmacol. (2020) 72:113343. doi: $10.1111 /$ jphp. 13286

51. Han XM, Qin YJ, Zhu Y, Zhang XL, Wang NX, Rang Y, et al. Development of an underivatized LC-MS/MS method for quantitation of 14 neurotransmitters in rat hippocampus, plasma and urine: application to CUMS induced depression rats. J Pharm Biomed Anal. (2019) 174:68395. doi: 10.1016/j.jpba.2019.06.043

52. Kim YK, Kim OY, Song J. Alleviation of depression by glucagon-like peptide 1 through the regulation of neuroinflammation, neurotransmitters, neurogenesis, and synaptic function. Front Pharmacol. (2020) 11:1270. doi: $10.3389 /$ fphar.2020.01270

53. Kowalczyk J, Nakos-Bimpos M, Polissidis A, Dalla C, Kokras N, SkalickaWozniak $\mathrm{K}$, et al. Xanthotoxin affects depression-related behavior and neurotransmitters content in a sex-dependent manner in mice. Behav Brain Res. (2021) 399:112985. doi: 10.1016/j.bbr.2020.112985

54. Smith GS, Oeltzschner G, Gould NF, Leoutsakos JS, Nassery N, Joo JH, et al. Neurotransmitters and neurometabolites in late-life depression: a preliminary magnetic resonance spectroscopy study at 7T. J Affect Disord. (2021) 279:41725. doi: $10.1016 /$ j.jad.2020.10.011

55. Liu C, Wang J, Xu S, An S, Tang S, He J, et al. Paecilomyces tenuipes extract prevents depression-like behaviors in chronic unpredictable mild stress-induced rat model via modulation of neurotransmitters. Mol Med Rep. (2017) 16:2172-8. doi: 10.3892/mmr.2017.6807

56. Xu H, Wang Z, Zhu L, Sui Z, Bi W, Liu R, et al. Targeted neurotransmitters profiling identifies metabolic signatures in rat brain by LC-MS/MS application in insomnia, depression and Alzheimer's disease. Molecules. (2018) 23:2375. doi: 10.3390/molecules23092375

57. Amin FU, Shah SA, Kim MO. Glycine inhibits ethanol-induced oxidative stress, neuroinflammation and apoptotic neurodegeneration in postnatal rat brain. Neurochem Int. (2016) 96:1-12. doi: 10.1016/j.neuint.2016.04.001

58. Gao L, Zhou F, Wang KX, Zhou YZ, Du GH, Qin XM. Baicalein protects PC12 cells from A $325-35$-induced cytotoxicity via inhibition of apoptosis and metabolic disorders. Life Sci. (2020) 248:117471. doi: 10.1016/j.lfs.2020.117471

59. Yan JJ, Du GH, Qin XM, Gao L. Baicalein attenuates the neuroinflammation in LPS-activated BV-2 microglial cells through suppression of pro-inflammatory cytokines, COX2/NF- $\mathrm{kB}$ expressions and regulation of metabolic abnormality. Int Immunopharmacol. (2020) 79:106092. doi: 10.1016/j.intimp.2019.106092

60. Liu R, Liao XY, Pan MX, Tang JC, Chen SF, Zhang Y, et al. Glycine exhibits neuroprotective effects in ischemic stroke in rats through the inhibition of

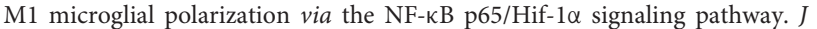
Immunol. (2019) 202:1704-14. doi: 10.4049/jimmunol.1801166

61. Wang $\mathrm{M}$, Gui $\mathrm{X}, \mathrm{Wu} \mathrm{L}$, Tian S, Wang $\mathrm{H}$, Xie L, et al. Amino acid metabolism, lipid metabolism, and oxidative stress are associated 
with post-stroke depression: a metabonomics study. BMC Neurol. (2020) 20:250. doi: 10.1186/s12883-020-01780-7

62. Jung J, Lee SM, Lee MJ, Ryu JS, Song JH, Lee JE, et al. Lipidomics reveals that acupuncture modulates the lipid metabolism and inflammatory interaction in a mouse model of depression. Brain Behav Immun. (2021) 94:424-36. doi: 10.1016/j.bbi.2021.02.003

63. Barch DM, Shirtcliff EA, Elsayed NM, Whalen D, Gilbert K, Vogel AC, et al. Testosterone and hippocampal trajectories mediate relationship of poverty to emotion dysregulation and depression. Proc Natl Acad Sci U S A. (2020) 117:22015-23. doi: 10.1073/pnas.2004363117

64. Fei W, Hou Y, Yue N, Zhou X, Wang Y, Wang L, et al. The effects of aqueous extract of Maca on energy metabolism and immunoregulation. Eur J Med Res. (2020) 25:24. doi: 10.1186/s40001-020-00420-7

65. Islam MR, Ali S, Karmoker JR, Kadir MF, Ahmed MU, Nahar Z, et al. Evaluation of serum amino acids and non-enzymatic antioxidants in drug-naïve first-episode major depressive disorder. BMC Psychiatry. (2020) 20:333. doi: 10.1186/s12888-020-02738-2

66. Rosário PW, Batista KC, Calsolari MR. Radioiodine-induced oxidative stress in patients with differentiated thyroid carcinoma and effect of supplementation with vitamins $\mathrm{C}$ and $\mathrm{E}$ and selenium (antioxidants). Arch Endocrinol Metab. (2016) 60:328-32. doi: 10.1590/2359-3997000000128

67. Duman RS, Aghajanian GK, Sanacora G, Krystal JH. Synaptic plasticity and depression: new insights from stress and rapid-acting antidepressants. Nat Med. (2016) 22:238-49. doi: 10.1038/nm.4050
68. Ren F, Guo R. Synaptic microenvironment in depressive disorder: insights from synaptic plasticity. Neuropsychiatr Dis Treat. (2021) 17:15765. doi: $10.2147 / \mathrm{NDT} . S 268012$

69. Chesworth R, Long LE, Weickert CS, Karl T. The endocannabinoid system across postnatal development in transmembrane domain neuregulin 1 mutant mice. Front Psychiatry. (2018) 9:11. doi: 10.3389/fpsyt.2018.00011

Conflict of Interest: The authors declare that the research was conducted in the absence of any commercial or financial relationships that could be construed as a potential conflict of interest.

Publisher's Note: All claims expressed in this article are solely those of the authors and do not necessarily represent those of their affiliated organizations, or those of the publisher, the editors and the reviewers. Any product that may be evaluated in this article, or claim that may be made by its manufacturer, is not guaranteed or endorsed by the publisher.

Copyright $\odot 2021$ Duan, Qiu, Bai, Qiao, Su, Lo, Lu, Xu, Wang, Li and Mo. This is an open-access article distributed under the terms of the Creative Commons Attribution License (CC BY). The use, distribution or reproduction in other forums is permitted, provided the original author(s) and the copyright owner(s) are credited and that the original publication in this journal is cited, in accordance with accepted academic practice. No use, distribution or reproduction is permitted which does not comply with these terms. 